\title{
L'acquisition de la temporalité en FRL2 : étude descriptive de narrations au passé par des apprenants néerlandophones et hispanophones
}

\author{
Hadermann, Pascale \& Ruyffelaert, Ariane \\ Université de Gand - Département de Linguistique \\ Pascale.Hadermann@ugent.be, Ariane.Ruyffelaert@ugent.be
}

\section{Introduction}

Les langues naturelles ont développé un vaste répertoire de moyens pour exprimer la temporalité, tels que les catégories verbales du temps et de l'aspect, les caractéristiques sémantiques temporelles inhérentes au verbe (et à ses compléments), les expressions périphrastiques du type commencer $\grave{a}$, continuer $\grave{a}$, se mettre à, être en train de + infinitif, des compléments adverbiaux exprimant le temps, la durée et des principes d'organisation discursive comme le Principe de l'Ordre Naturel (voir entre autres Klein 1994). Confronté avec cette kyrielle de moyens linguistiques, tout locuteur aura un long trajet à parcourir avant d'arriver à la maîtrise complète de l'expression de la temporalité et ceci est sans doute d'autant plus vrai lorsqu'il s'agit de s'approprier ce système dans une langue étrangère qui est typologiquement distincte de la langue source (voir entre autres Housen 2002 et Izquierdo \& Collins 2008 pour un commentaire sur les « transferts négatifs » et Salaberry 2005 sur le « transfert positif » en cas de ressemblances entre langues source et cible).

Les temps verbaux constituent un des paradigmes qui se prêtent le mieux à l'observation des divergences intersystémiques et de leur impact au niveau de l'expression de la temporalité. Pour comprendre les processus acquisitionnels de ces temps verbaux et l'éventuel effet de la proximité entre langues source et cible, il est intéressant de comparer des interlangues d'apprenants ayant des langues sources issues de familles linguistiques différentes (par ex. le néerlandais et l'espagnol) mais dont une appartient au même groupe que la langue cible (par ex. le français, langue romane tout comme l'espagnol). Il se pourrait que, pour les apprenants hispanophones, l'acquisition des temps verbaux se déploie autrement car, à quelques exceptions près, le système verbal de l'espagnol est très proche de celui du français (Noyau \& Vasseur 1986, Starren 2001, Benazzo 2007, Amenós 2010).

L'objectif de notre contribution est d'analyser comment des apprenants néerlandophones et hispanophones du français langue étrangère (FR L2) acquièrent la temporalité - plus spécifiquement la référence au passé - et de vérifier dans quelle mesure leurs interlangues se rapprochent de la langue cible ou, au contraire, reflètent de manière plus ou moins fidèle des mécanismes propres à la langue maternelle (L1). Afin de dresser une image aussi complète que possible, nous présenterons d'abord brièvement les notions essentielles à notre étude $(\S 2)$ ainsi que nos hypothèses de recherche et nos données $(\S 3)$. Nous adopterons, dans notre description, une approche multifactorielle qui intègre l'analyse de différents moyens linguistiques - morphologiques, lexicaux, sémantiques ${ }^{1}$ - et qui essaiera de déterminer la raison du choix pour tel ou tel temps verbal du passé (§4). Pour expliquer ces choix possibles, nous vérifierons les hypothèses de la primauté de l'aspect lexical (\$5) et du transfert de la L1 (§6). 


\title{
2 Cadre
}

\subsection{L'expression du temps}

\subsubsection{Le temps verbal et le temps référentiel}

Le temps verbal décrit «l'orientation d'un procès par rapport à un repère » sur un axe orienté du passé vers l'avenir (Wilmet 2007 : 68). Il établit un rapport entre le moment de la parole (désormais MP) et le temps de la situation exprimée par le verbe (désormais TSit) qui est de l'ordre de la simultanéité, de l'antériorité ou de la postériorité. Il se peut que la situation exprimée par le verbe se rapporte à un autre point sur l'axe temporel que le MP, ce qui donne lieu à l'identification d'un nouveau repère que Reichenbach (1966) appelle the point of reference ou " the temporal standpoint from which the speaker invites his audience to consider the occurrence of the event (or the obtaining state) » (Taylor 1977: 203, cité par Kihlstedt 1998 : 24). Nous le désignerons par le terme de temps référentiel (TRé).

\subsubsection{L'aspect grammatical et le mode d'action}

L'aspect grammatical indique la façon dont l'action se déroule, indépendamment du MP. Il concerne la perspective que le sujet parlant adopte par rapport au déroulement, à la progression ou à l'accomplissement du procès. Etant donné la richesse de ces perspectives, plusieurs classifications et étiquettes ont été proposées, selon que l'on envisage (i) le regard du locuteur vis-à-vis du procès : soit de l'extérieur, soit de l'intérieur (par ex. aspect global vs sécant, Wilmet 2007 : 71), (ii) la pertinence ou l'impertinence du procès par rapport au présent (par ex. aspect parfait vs aoriste, Kihlstedt 1998), (iii) la vision résultative ou non du procès (par ex. aspect perfectif vs imperfectif, Populier 1985).

De plus, toute prédication verbale véhicule un sens aspectuel inhérent que l'on nomme habituellement « aspect lexical » ou « mode d'action » et qui ne doit pas être confondu avec l'aspect grammatical.

\begin{abstract}
« La catégorie de l'aspect se décompose en aspect lexical [= mode d'action] et aspect grammatical. L'aspect lexical correspond au type de procès (activité, état, accomplissement, ...) exprimé par le lexème verbal et son environnement actanciel (par exemple, on établit au moyen de tests syntaxiques que manger du poulet constitue une 'activité'). L'aspect grammatical définit le mode de présentation du procès (accompli, inaccompli, itératif, ...) tel qu'il est indiqué essentiellement par les marques grammaticales (temps morphologiques, semi-auxiliaires, adverbes d'aspect. » (Gosselin 1996 : 10)
\end{abstract}

Vendler (1967), pionnier dans le domaine de la définition et de la classification des modes d'action, distingue quatre groupes de verbes d'après leur sens aspectuel inhérent :

a. les verbes d'état qui se caractérisent par une idée durative (p. ex. être, avoir, aimer) ; ils impliquent une continuation de la situation décrite sans changement d'état; ils sont non dynamiques et puisqu'ils ne tendent pas vers une fin, ils sont également considérés comme atéliques ;

b. les verbes d'activité où chaque partie du procès est de même nature que le tout (p. ex. courir, jouer, naviguer); ils sont dynamiques et exigent de l'énergie pour se réaliser et pour faire perdurer le procès; ils n'envisagent pas la fin du procès, ils sont donc atéliques ;

c. les verbes d'accomplissement dont le procès s'achemine inévitablement vers un terme (p. ex. manger une pomme, courir cinq kilomètres ${ }^{2}$ ); dans ce cas, il faut de l'énergie non seulement pour continuer les procès exprimés, mais aussi pour les faire naître ou pour les faire aboutir ; ils sont téliques ;

d. les verbes d'achèvement qui, à cause de leur caractère instantané, réfèrent au terme (ou à l'origine) du cheminement (p. ex. reconnaître, entrer, tomber) ; ils sont également téliques et, en plus, ils possèdent le trait [+ponctuel] lié à l'expression de l'instantanéité ${ }^{3}$. 
Le tableau 1 résumé ce jeu sur les traits dynamique, télique et ponctuel.

\begin{tabular}{|c|c|c|c|c|}
\hline & état & activité & accomplissement & achèvement \\
\hline dynamique & - & + & + & + \\
télique & - & - & + & + \\
ponctuel & - & - & - & + \\
\hline
\end{tabular}

Tableau 1. Les traits sémantiques des modes d'action, d'après Andersen (1991)

Même si le mode d'action et l'aspect grammatical sont des notions distinctes, ils paraissent parfois intimement liés. Ainsi, les verbes d'état et d'activité possèdent certaines affinités avec l'aspect imperfectif - ex. 1 - et il est généralement admis que les verbes d'accomplissement et d'achèvement s'accordent bien avec l'aspect perfectif - ex. 2. Mais ce parallélisme n'est pas absolu. En 3 par exemple, le verbe d'activité préparer reçoit le trait [+télique] : l'aspect perfectif focalise les bornes du procès et circonscrit sa durée temporelle. De même, en 4 tomber est marqué par l'aspect imperfectif : l'imparfait ajoute ici un effet de sens à la prédication, c'est-à-dire un ralentissement (Bergström 1995) ou une expansion (Labeau 2005).

1. Les pirates se rapprochaient de plus en plus, la Licorne sentait la menace s'approcher. [CCF.5] (verbe d'activité et aspect imperfectif)

2. Ils ancrèrent le navire près des côtes. [CCF.1] (verbe télique et aspect perfectif)

3. Alors, l'équipage de La Licorne prépara sa défense en armant les canons. [CCF.1] (verbe d'activité et aspect perfectif)

4. Le soir tombait, les pirates fêtaient bruyamment leur victoire à grands verres de rhum... [CCF.4] (verbe d'achèvement et aspect imperfectif)

Grâce à l'interaction entre le temps, l'aspect et le mode d'action, le locuteur a donc à sa disposition un large éventail de possibilités d'expression ${ }^{4}$. Dans ce qui suit, nous présenterons les différences essentielles dans l'exploitation de ces possibilités entre le français et le néerlandais (\$2.2) ainsi que les difficultés qu'elles posent au niveau des interlangues (\$2.3).

\subsection{La référence au passé dans le récit en français et en néerlandais L1}

Weinrich (1973 : 115) distingue, comme deux lois fondamentales de la narrativité, un premier plan ${ }^{5}$ et un arrière-plan :

«[L]e premier plan [est] habituellement ce pourquoi l'histoire est racontée ; ce que retient un compte rendu, factuel; ce que le titre résume ou pourrait résumer; ou encore, ce qui, au fond, donne aux gens l'envie de délaisser un instant leurs occupations pour écouter une histoire si étrangère à leur univers quotidien ; $[. .$.$] à$ l'inverse, l'arrière-plan du récit: dans son sens le plus large, c'est ce qui lui seul n'éveillerait pas l'intérêt, mais qui aide l'auditeur à s'orienter à travers le monde raconté et lui rend l'écoute plus aisée. »

et Wallace (1982 : 209, cité par Labelle 1987 : 12) d'ajouter :

«Si une langue offre un contraste entre un aspect perfectif et d'autres aspects, alors une partie du sens de l'aspect perfectif, du moins dans la narration, est de décrire les événements principaux, séquentiels, du premier plan, tandis qu'une partie du sens de l'aspect non perfectif, et spécialement de l'imperfectif, est de donner des renseignements supplémentaires d'arrière-plan. » 


\subsubsection{La référence au passé en français}

Les cinq temps verbaux majoritairement présents dans le corpus de contrôle francophone sont le passé composé, l'imparfait, le passé simple, le présent historique et le plus-que-parfait qui, même s'ils expriment tous l'antériorité du TSit par rapport au MP, se distinguent l'un de l'autre par leurs valeurs temporelle (la relation entre le TSit et le MP) et aspectuelle (la relation entre le TSit et le TRé). En outre, le type de discours et la mise en relief du récit (l'avant-plan $v s$ l'arrière-plan) constituent deux paramètres importants pour la description des temps verbaux en français.

Weinrich (1973) et Labelle (1987) ont mis en relief la position ambiguë du passé composé (désormais PC) : en tant que temps composé, il marque un aspect résultatif (TRé > TSit) mais il garde un lien avec le présent. Son sens «prototypique » est celui de parfait : il indique que le TSit est antérieur par rapport au TRé, qui correspond au MP. Ainsi, il décrit la situation résultant du procès 5 mais il admet également un sens dérivé, celui d'aoriste, qui exprime un TRé antérieur au MP. Imbs (1960) observe que dans ce cas, il est accompagné obligatoirement d'une expression temporelle 6 .

5. Jean-Baptiste a guéri.

6. Fiorine s'est endormie (TSit) à huit heures du soir (= TRé).

Quant à l'imparfait (désormais IMP), il sert avant tout à exprimer l'aspect imperfectif : le locuteur ne prend pas en considération l'intervalle entier qui correspond au TSit mais se limite à constater ce qui se passe au moment pris comme repère, le TRé, excluant ainsi le début ou la fin de l'intervalle. L'IMP situe le TRé, qui est inclus dans le TSit, avant le MP. Il fonctionne comme un temps coréférentiel de l'arrièreplan qui décrit des actions simultanées aux événements narratifs. Cependant, d'après Hopper (1979), le sens premier de l'IMP ne serait pas de donner des renseignements d'arrière-plan : c'est parce qu'il aurait un sens descriptif qu'il serait capable de fournir des informations d'arrière-plan.

Le passé simple (désormais PS) se distingue de l'IMP par sa valeur aspectuelle perfective (TSit $\in$ TRé). De même que le $\mathrm{PC}$, il présente le procès exprimé par le verbe comme un tout inanalysable. Il représente le temps du récit et il permet de se distancier complètement du MP (Delatour et alii 1991). Ce temps verbal n'exprime jamais l'aspect parfait, comme le fait le $\mathrm{PC}^{6}$. Ceci explique son absence dans la langue parlée et son emploi fréquent à la troisième personne. Sa fonction de base est de nature aoriste. Il situe le procès au passé, et plus précisément au passé lointain, et donne au locuteur l'occasion de présenter les actions multiples comme successives. Le PS sert donc à indiquer l'avant-plan de la narration, comme le permet également l'emploi du présent historique.

Judge (2002 : 138) décrit le présent historique de manière suivante :

«Pour certains, c'est un présent fictif qui décrit les événements comme s'ils se déroulaient devant les yeux de celui qui écrit, pour d'autres, il est devenu un temps 'passe-partout', une forme neutre dont la valeur dépend uniquement du contexte. »

Le critère de l'alternance possible avec un autre temps au passé permettrait de distinguer le présent historique à l'intérieur du récit d'un présent atemporel ou d'un présent " par défaut ».

Enfin, la valeur de base du plus-que-parfait (désormais $\mathrm{PQP}$ ) est celle d'antériorité : il situe le TRé après le TSit (TRé > TSit), ce qui lui permet d'exprimer un aspect résultatif, comme le PC aux fonctions parfait et aoriste.

\subsubsection{La référence au passé en néerlandais}

En néerlandais, l'imperfectum est le temps du récit qui permet de faire avancer l'histoire. Il traduit aussi bien l'avant-plan que l'arrière-plan et peut exprimer à la fois un aspect perfectif et un aspect imperfectif. L'imparfait en français, par contre, n'intervient pas pour faire avancer l'histoire dans le temps. En tant que temps coréférentiel, il occupe une fonction essentiellement descriptive dans le récit. Il ajoute des informations complémentaires qui se déroulent simultanément par rapport à l'action principale, exprimée par le passé simple, le passé composé ou le présent historique. 
Le perfectum néerlandais correspond morphologiquement au passé composé : il se construit à l'aide d'un auxiliaire et d'un participe passé. Comme il alterne facilement avec l'imperfectum, le néerlandais l'exploite peu dans les récits, contrairement au français où le passé composé intervient à l'avant-plan de l'histoire et présente les événements dans un ordre successif, capacités qu'il partage avec le passé simple. Ce dernier temps est uniquement attesté dans le corpus des natifs et, pour cette raison, nous n'en traiterons pas à part ${ }^{7}$.

Pour ce qui est du praesens historicum et du présent historique ainsi que du plusquamperfectum et du plus-que-parfait, les emplois semblent similaires dans les deux langues.

\subsection{La référence au passé dans les interlangues}

Dans le domaine de l'apprentissage des langues étrangères, plusieurs recherches ont été dédiées à la référence au passé. Celle-ci se révèle d'autant plus intéressante que les systèmes des langues sources et cibles ne sont pas nécessairement convergents, ce qui est le cas, comme nous venons de le voir, du couple néerlandais-français contrairement au couple espagnol-français.

Ainsi, Van der Linden (2000 : 235-236) relie les difficultés qu'éprouvent ses apprenants néerlandophones pré-avancés au niveau de la temporalité en français - avec un suremploi de l'imparfait -, aux divergences entre les systèmes des langues source et cible. De même, Starren (2001), dans son étude sur l'emploi des temps chez des apprenants turcs et marocains du néerlandais et du français, souligne l'importance des interférences. Elle montre que, dans les cas où la langue maternelle se sert par exemple d'adverbes pour marquer le temps au lieu de marques morphosyntaxiques, l'apprenant cherchera des moyens comparables dans la langue seconde (Starren 2001 : 6). Or, elle note également que dans une phase ultérieure l'insertion de ces adverbes ou formes équivalentes peut exercer une influence positive et fonctionner comme « un moule » pour la construction d'un système morphologique approprié (Starren 2001 : 254).

Parallèlement à ces observations, Izquierdo (2009 : 588) met en évidence la possibilité de transferts positifs de la L1 vers la L2 dans le cas de ressemblances ou d'analogies fonctionnelles ou formelles : ses apprenants hispanophones, qui disposent, dans leur L1, d'un système de référence au passé plus ou moins analogue au français, ne sur-emploient pas le passé composé, contrairement aux apprenants anglophones qui n'ont pas, dans leur L1, un temps équivalant à l'imparfait (voir aussi Howard 2002 et 2012, de même que Paprocka-Piotrowska 2002 pour des polonophones, Schlyter 1996 et Lindgren 2008 pour des suédophones).

«Dans leur L1, les apprenants hispanophones font, de manière systématique, une différenciation entre les valeurs de l'habitude, de la répétition, de la progression et de la continuité de l'imparfait. Ils font aussi une différenciation entre ces valeurs et la valeur aspectuelle du passé composé. Il est possible que les représentations internes que les hispanophones possèdent du sens et de la forme des aspects grammaticaux dans leur L1 facilitent la compréhension, la rétention et l'accès aux valeurs aspectuelles des formes langagières équivalentes dans la L2. Il est possible également que ces représentations contribuent à éviter le confinement de l'imparfait à une seule valeur aspectuelle, comme il semble se produire chez les apprenants qui n'ont pas l'imparfait dans leur L1. » (Izquierdo 2009 : 604-5)

\subsection{Bilan}

La temporalité dans le domaine du verbe ne se laisse pas appréhender de manière univoque : elle se manifeste au niveau morphologique par le choix du temps et de l'aspect grammatical, au niveau sémantique par les propriétés inhérentes au sens du verbe, au niveau lexical par l'emploi d'expressions temporelles pour expliciter la situation du procès exprimé par le verbe dans le temps. Avant d'examiner en $(\S 4)$ comment ces trois niveaux sont pris en compte dans deux types d'interlangue différents et de vérifier l'influence d'analogies entre langues source et cible à un certain stade de l'apprentissage, nous présenterons en $(\S 3)$ nos hypothèses de recherche ainsi que nos corpus. 


\section{Hypothèses, corpus et méthodologie}

\subsection{Questions et hypothèses de recherche}

Nos deux questions de recherche principales - c'est-à-dire les questions de savoir (i) comment des apprenants néerlandophones et hispanophones expriment la référence au passé en FLE et (ii) dans quelle mesure il se dégage des analogies entre les moyens mis en œuvre par les deux groupes d'apprenants seront abordées de manière pluridimensionnelle. Afin d'apporter des éléments de réponse, nous vérifierons les moyens morphologiques utilisés pour situer les événements dans le temps, nous analyserons l'emploi d'expressions temporelles dans les récits, nous creuserons l'effet du mode d'action sur les choix opérés par les apprenants et enfin nous comparerons les résultats obtenus pour les deux groupes.

Des deux grandes questions ci-dessus découlent les sous-questions suivantes et leurs hypothèses associées :

a. Question 1: Les apprenants emploient-ils les temps du passé dans leurs narrations et si oui, lesquels?

- Hypothèse : nous nous attendons à ce qu'ils recourent à des temps tels que l'imparfait et le passé composé pour rédiger une narration au passé et que chez les néerlandophones il se manifeste une tendance à privilégier l'imparfait (conformément à ce qu'a observé Van der Linden 2000) alors que chez les hispanophones il n'y aurait pas d'anomalies (cf. Izquierdo 2009).

b. Question 2: Les apprenants associent-ils correctement temps verbal et expression temporelle de type adverbial?

- Hypothèse : nous nous attendons à ce que nos apprenants de niveau post-basique à intermédiaire ${ }^{8}$ combinent correctement ces deux moyens, morphologique et lexical (cf. Starren 2001).

c. Question 3: Les apprenants s'appuient-ils avant tout sur le mode d'action pour choisir un temps verbal?

- Hypothèse : nous nous attendons en effet à ce que le mode d'action joue un rôle crucial dans le choix du temps verbal (cf. entre autres Starren 2001).

Les questions (1) et (3) nous amèneront à reprendre plus en détail l'hypothèse de la primauté de l'aspect lexical ${ }^{9}$ en (§5) et l'hypothèse du transfert (cf. supra : §2.3) en (§6).

\subsection{Corpus}

Notre étude de la référence au passé en FR L2 s'appuie sur deux corpus de narrations écrites respectivement par vingt apprenants de la $4^{\text {ème }}$ année de l'enseignement secondaire général en Flandre (NL FR L2) et par quinze apprenants de la $3^{\text {ème }}$ année de l'enseignement secondaire général en Espagne (ESP FR L2). Il s'agit plus précisément d'apprenants qui, en Flandre, suivent la filière «Latin ou Grec/Mathématiques » avec un enseignement de français à raison de 3h/semaine et qui, en Espagne, ont un enseignement de français à raison de $2 \mathrm{~h} /$ semaine. Les élèves flamands sont âgés de 15 à 17 ans et les élèves espagnols ont entre 14 et 15 ans ${ }^{10}$.

Pour notre enquête, ils ont rédigé en classe une histoire à partir de dix-huit images tirées de la bande dessinée Le Secret de la Licorne d'Hergé ${ }^{11}$. Ils ont pu travailler pendant une demi-heure à leur texte qui devrait avoir une longueur minimale d'une page. Les élèves flamands avaient préalablement bénéficié d'un cours sur les contextes d'emploi du passé composé et de l'imparfait et les hispanophones avaient eu des cours sur la formation et l'emploi du passé composé. Pour attirer l'attention sur la référence au passé, l'enquêtrice a insisté sur le temps où se déroule l'histoire de la Licorne (1698), comme le précise le capitaine Hadoque dans la première bulle : 
« Je souhaite vous raconter l'histoire de La Licorne, un bateau du temps de Louis XIV, qui avait quitté les Antilles en 1698 avec à bord beaucoup d'épices et surtout du rhum. »

Outre ces deux corpus d'apprenants, nous disposons de trois corpus de contrôle pour le français ( 9 textes, appelé $\mathrm{CCF}$ ), pour le néerlandais ( 8 textes, $\mathrm{CCN}$ ) et pour l'espagnol (4 textes, CCESP), rédigés par des locuteurs natifs adolescents et adultes ${ }^{12}$. Le tableau ci-dessous en présente les détails.

\begin{tabular}{|l|c|c|c|c|c|}
\hline & NL FR L2 & ESP FR L2 & FR L1 & NL L1 & ESP L1 \\
\hline Nombre de textes & 20 & 15 & 9 & 8 & 4 \\
\hline Nombre de verbes finis & 617 & 343 & 246 & 194 & 111 \\
\hline Moyenne de verbes/texte & 31 & 23 & 27 & 24 & 28 \\
\hline
\end{tabular}

Tableau 2. Présentation des cinq corpus

\subsection{Méthodologie}

Pour l'analyse des moyens morphologiques, chaque verbe fini a été codé d'après le temps utilisé et d'après le degré de correction ( \pm correct avec, dans le cas d'erreurs, une distinction entre erreur formelle, fonctionnelle ou formelle et fonctionnelle).

7. Mais les pirates ont approché La Licorne et le capitaine n'avait pas vu. [4.11] $]^{13}$ Le premier prédicat est formellement incorrect (approcher pour se rapprocher) et le second présente une erreur fonctionnelle (avait $v u$ au lieu de $a v u$ ).

8. Ils sont revenus immédiatement et ils climbaient sur le navire. [4.10] Erreur formelle et fonctionnelle : climbaient au lieu de ont grimpé.

Au niveau lexical, quatre types d'expressions temporelles (TA) ont été pris en considération : les marqueurs de position (TAP), de durée (TAD), de fréquence (TAF) et de contraste (TAC). Il s'agit d'adverbes ou de locutions adverbiales et de syntagmes nominaux, accompagnés ou non d'une préposition de temps. Nous avons comptabilisé toutes les occurrences de ces TA en combinaison avec des formes verbales au passé composé, à l'imparfait et au plus-que-parfait.

Quant à l'analyse des moyens sémantiques, nous avons identifié le mode d'action des syntagmes à verbe fini en nous appuyant entre autres sur les tests de la dynamicité et de l'agentivité tels qu'ils se trouvent présentés et résumés dans les études de Bergström (1995) et de Gosselin \& François (1991).

Dans ce qui suit, nous décrirons les résultats obtenus par ces méthodes de classification, sous forme d'effectifs réels (N) accompagnés éventuellement du pourcentage pour mettre en évidence la proportionnalité des emplois.

\section{La référence au passé en FR L2}

\subsection{Les moyens morphologiques}

\begin{tabular}{|l|c|c|c|c|}
\hline & \multicolumn{2}{|c|}{ NL FR L2 } & \multicolumn{2}{c|}{ ESP FR L2 } \\
\hline Emploi des temps & $\mathrm{N}$ & $\%$ & $\mathrm{~N}$ & $\%$ \\
\hline PC (PS en FR L1/ESP L1) & 116 & 18,8 & 194 & $\mathbf{5 6 , 6}$ \\
\hline IMP & 298 & $\mathbf{4 8 , 3}$ & 29 & 8,5 \\
\hline
\end{tabular}




\begin{tabular}{|l|c|c|c|c|}
\hline PR & 156 & 25,3 & 115 & $\mathbf{3 3 , 5}$ \\
\hline PQP & 34 & 5,5 & 0 & 0 \\
\hline Autres $^{14}$ & 13 & 2,1 & 5 & 6,8 \\
\hline Total & 617 & 100 & 343 & 100 \\
\hline
\end{tabular}

Tableau 3. Emploi des temps en FR L2 par des apprenants NL et ESP (nombres \& \%)

L'imparfait est le temps verbal préféré de la plupart de nos apprenantes néerlandophones. En second lieu vient la forme verbale à l'indicatif présent ; le passé composé occupe la troisième place. Cette image n'est pas identique à celle qui se dégage du corpus des hispanophones qui préfèrent recourir au passé composé et au présent et qui semblent éviter l'imparfait. Alors que le suremploi de l'imparfait chez les néerlandophones correspond à nos attentes et confirme les résultats d'autres études, sa faible utilisation par les apprenants ESP est surprenante car dans leur L1 ce temps verbal est bel et bien productif. Ceci pourrait s'expliquer par le fait que les apprenants hispanophones (de la troisième année) se trouvent encore dans un stade acquisitionnel inférieur aux apprenants néerlandophones, à savoir le stade postinitial dans lequel l'emploi correct de l'imparfait ne se manifeste pas encore (cf. Bartning \& Schlyter 2004).

Un autre fait inattendu est le recours relativement fréquent au présent dans les deux corpus. La question qui devrait donc d'abord être posée est de savoir avec quelle valeur ce présent s'emploie : valeur de présent, substitut de l'imparfait ou du passé composé ${ }^{15}$.

\begin{tabular}{|l|c|c|}
\hline PRESENT pour ... (nombres) & NL FR L2 & ESP FR L2P \\
\hline ... présent (PRES) & 17 & 15 \\
\hline .. passé composé (PC) & $\mathbf{8 7}$ & $\mathbf{4 8}$ \\
\hline ... imparfait (IMP) & 52 & $\mathbf{5 2}$ \\
\hline total & 156 & 115 \\
\hline
\end{tabular}

Tableau 4. Distribution des formes verbales au présent

Les présents à valeur présent ne sont pas très fréquents; ils apparaissent surtout au début des textes où ils servent à introduire le récit 9-11 ou à donner un commentaire sur l'événement 10-12.

9. Nous nous trouvons aux Antilles en 1698, l'époque de Louis XIV. [4.3]

10. Maintenant, il n'est toujours pas oublié c'est un héro pour tous les autres capitaines. [4.3]

11. Dans le premier designe se trouve le capitan Hadock qui est raconte a Tintin une histoire de son grand-père. [3.8]

12. Dans la première image le capitaine Haddock parle avec Tintin, il raconte une histoire de 1698. [3.11]

Le présent pour passé endosse tantôt les valeurs du PC, tantôt celles de l'IMP mais dans la majorité des cas, il semble équivaloir à un PC chez les apprenants NL et à un IMP chez les apprenants ESP. Il arrive qu'il se substitue, à l'intérieur d'une même phrase aussi bien au PC qu'à l'IMP.

13. Les deux capitaines sont très fort mais le parent perds et les pirates lui attachent à un pillier. [4.1]

Les deux capitaines étaient très fort mais le parent a perdu et les pirates lui ont attaché à un pillier.

Le présent fonctionne donc la plupart du temps comme une stratégie d'évitement, qui permet de contourner l'emploi formel et/ou fonctionnel plus complexe d'un temps verbal du passé. 


\begin{tabular}{|c|c|c|c|c|}
\hline & \multicolumn{2}{|c|}{ NL FR L2 } & \multicolumn{2}{|c|}{ ESP FR L2 } \\
\hline & $\mathrm{N}$ & $\%$ & $\mathrm{~N}$ & $\%$ \\
\hline passé composé & 116 & 100 & 194 & 100 \\
\hline correct & 93 & 80,2 & 115 & 59,3 \\
\hline incorrect & 23 & 19,8 & 79 & 40,7 \\
\hline * forme & 18 & 78,3 & 28 & 35,4 \\
\hline * fonction & 4 & 17,4 & 33 & 41,8 \\
\hline * forme et fonction & 1 & 4,3 & 18 & 22,8 \\
\hline imparfait & 298 & 100 & 29 & 100 \\
\hline correct & 112 & 37,6 & 10 & 34,5 \\
\hline incorrect & 186 & 62,4 & 19 & 65,5 \\
\hline * forme & 21 & 11,3 & 3 & 15,8 \\
\hline * fonction & 141 & 75,8 & 0 & 0 \\
\hline * forme et fonction & 24 & 12,9 & 16 & 84,2 \\
\hline présent & 156 & 100 & 115 & 100 \\
\hline correct & 14 & 9 & 10 & 4,5 \\
\hline incorrect & 142 & 91 & 105 & 91,3 \\
\hline * forme & 1 & 0,7 & 8 & 7,6 \\
\hline * fonction & 124 & 87,3 & 32 & 30,5 \\
\hline * forme et fonction & 17 & 12 & 65 & 61,9 \\
\hline
\end{tabular}

Tableau 5. Aperçu de la correction formelle et fonctionnelle des temps verbaux

Le tableau 5 illustre le degré de correction pour le passé composé, l'imparfait et le présent. Seul le PC atteint un taux de plus de 50\% d'emplois corrects dans les deux corpus mais les néerlandophones semblent mieux maîtriser les valeurs intrinsèques au PC, les fautes étant essentiellement de type formel 14 alors que chez les hispanophones elles sont de type fonctionnel 15.

14. Ils se sont assis dans le jardin et il est commencé à parler. [4.7]

15. Quand les pirates ont été plus pres, ils ont lancé un canon. [3.4]

Des deux autres temps verbaux, l'emploi du PRES s'avère le plus problématique. Les erreurs sont essentiellement dues à un emploi fonctionnellement incorrect dans les deux corpus : le PRES relève en effet d'une stratégie d'évitement pour l'IMP et pour le PC (cf. supra). Quant à l'IMP, forme fréquente en NL FR L2, 62,4\% de ses occurrences sont incorrectes, avec des erreurs essentiellement fonctionnelles 16. Les mêmes tendances s'observent pour les rares attestations dans le corpus ESP FR L2.

$$
\text { 16. « Prendre le canon! » criait-il. [4.4] }
$$

Ces différents résultats suggèrent un lien remarquable entre les trois temps étudiés. Les emplois fonctionnellement erronés de l'IMP et du PRES par les apprenants NL et du PRES par les apprenants $\mathrm{ESP}^{16}$ pourraient être rapprochés des phénomènes de sous-emploi respectivement du PC et de l'IMP dans les deux corpus. Nous reviendrons sur cette question de sous-emploi dans la dernière partie de notre étude. 


\subsection{Les moyens lexicaux}

\begin{tabular}{|r|c|c|}
\hline & NL FR L2 & ESP FR L2 \\
\hline TA & 91 & $\mathbf{1 7}$ \\
\hline position & 46 & 8 \\
\hline durée & 30 & 9 \\
\hline fréquence & 5 & 0 \\
\hline contraste & 10 & 0 \\
\hline
\end{tabular}

Tableau 6. Distribution des expressions temporelles

Dans le corpus ESP FR L2 figurent très peu d'expressions temporelles et celles que nous avons relevées se répartissent équitablement sur les catégories de position 17 et de durée 18. Les apprenants hispanophones semblent préférer l'explicitation de la référence au temps à l'aide de connecteurs de balisage et plus spécifiquement le connecteur et 19, tendance qui se retrouve également chez les néerlandophones 20 .

17. Dans la nuit tout les pirates avons celebré qu'ils ont gagné avec whisky et beaucoup de rhum. [3.6]

18. Soudainement un homme a vu quelque chose dans la distance. [3.1]

19. Les pirates ont sauté dans l'interieur de La Licorne et ils ont pris ses épées pour attaquer les marines qui se trouvent dans le bateau. [3.1]

20. Mais un jour le père regarde à sa longue-vue et il voit un pavillon avec une tête de mort et il crie : «Des pirates »! [4.1]

Ce suremploi de et a également été observé dans d'autres études (cf. entre autres Paprocka-Piotrowska 2002). A côté de sa fonction principale de mot de liaison, il sert généralement à marquer une succession rapide d'événements tant aux niveaux intraphrastique qu'interphrastique.

Pour ce qui est des expressions temporelles proprement dites, nous avons déjà mentionné le lien qui s'instaurerait avec les moyens morphologiques. C'est ce que nous examinons à l'aide des résultats du tableau 7 qui reprend uniquement le corpus NL FR L2, faute de données représentatives pour les apprenants hispanophones. Nous avons extrait des occurrences du PC, de l'IMP et du PQP toutes celles qui sont accompagnées d'une TA dans la même proposition. En réunissant tous les temps verbaux qui sont fonctionnellement corrects d'une part, et toutes les formes verbales qui présentent une anomalie du point de vue fonctionnel d'autre part, nous obtenons la distribution suivante.

\begin{tabular}{|l|c|c|c|c|c|c|}
\hline & \multicolumn{3}{|c|}{$\mathrm{N}$} & \multicolumn{3}{c|}{$\%$} \\
\hline & total & correct & incorrect & total & correct & incorrect \\
\hline PC + TA & $17 / 116$ & 17 & 0 & 14,7 & 100 & 0 \\
\hline IMP + TA & $40 / 298$ & 15 & $\mathbf{2 5}$ & 13,4 & 37,5 & 62,5 \\
\hline PQP + TA & $8 / 34$ & 5 & 3 & 23,5 & 62,5 & 37,5 \\
\hline PC/IMP/PQP + TA & $65 / 448$ & 37 & 28 & 15 & 56,9 & 43,1 \\
\hline
\end{tabular}

Tableau 7. Interaction des moyens morphologiques et lexicaux NL FR L2

Les apprenants NL combinent toujours le PC avec l'expression temporelle appropriée 21 mais inversement celle-ci n'est pas capable de déclencher un emploi correct dans tous les cas de figure : dans 
25 exemples surgit un imparfait alors que l'expression temporelle pointe clairement vers le PC 21. Ces résultats ne confirment donc pas notre hypothèse du rôle déclencheur favorable qu'assumeraient les expressions temporelles.

21. Mon parent a immédiatement pris sa longue-vue et a aussi vu le pavillon avec la tête de mort. [4.12]

22. Donc la deuxième fois la vigie criait encore plus fort : « Attention, attention, des pirates, des pirates ! » [4.12]

\subsection{Les moyens sémantiques}

Enfin, il nous reste à examiner dans quelle mesure les apprenants se laissent influencer, dans leur emploi des temps, par le mode d'action exprimé par le syntagme verbal.

\begin{tabular}{|l|c|c|c|c|c|c|c|c|}
\hline \multirow{2}{*}{ NL FR L2 } & \multicolumn{2}{|c|}{ état } & \multicolumn{2}{c|}{ activité } & \multicolumn{2}{c|}{ accomplissement } & \multicolumn{2}{c|}{ achèvement } \\
\hline & $\mathrm{N}$ & $\%$ & $\mathrm{~N}$ & $\%$ & $\mathrm{~N}$ & $\%$ & $\mathrm{~N}$ & $\%$ \\
\hline passé composé & 8 & 4,1 & 28 & 22,6 & 15 & 20,5 & 65 & $\mathbf{3 3 , 3}$ \\
\hline imparfait & 132 & $\mathbf{6 7 , 7}$ & 60 & $\mathbf{4 8 , 4}$ & 40 & $\mathbf{5 4 , 8}$ & 66 & $\mathbf{3 3 , 8}$ \\
\hline plus-que-parfait & 0 & 0 & 8 & 6,5 & 3 & 4,1 & 23 & 11,8 \\
\hline FdB* pour PC & 15 & 7,7 & 23 & 18,5 & 14 & 19,2 & 35 & 17,9 \\
\hline FdB pour IMP & 40 & 20,5 & 5 & 4 & 1 & 1,4 & 6 & 3,1 \\
\hline total & 195 & 100 & 124 & 100 & 73 & 100 & 195 & 99,9 \\
\hline
\end{tabular}

* FdB, abréviation pour forme de base, désigne les emplois à défaut du présent

Tableau 8. Les modes d'action et leurs temps préférés en NL FR L2

\begin{tabular}{|l|c|c|c|c|c|c|c|c|}
\hline \multirow{2}{*}{ ESP FR L2 } & \multicolumn{2}{|c|}{ état } & \multicolumn{2}{c|}{ activité } & \multicolumn{2}{c|}{ accomplissement } & \multicolumn{2}{c|}{ achèvement } \\
\hline & $\mathrm{N}$ & $\%$ & $\mathrm{~N}$ & $\%$ & $\mathrm{~N}$ & $\%$ & $\mathrm{~N}$ & $\%$ \\
\hline passé composé & 24 & 32,9 & 102 & $\mathbf{6 9 , 4}$ & 27 & $\mathbf{6 2 , 8}$ & 41 & $\mathbf{6 8 , 3}$ \\
\hline imparfait & 12 & 16,4 & 11 & 7,5 & 1 & 2,3 & 5 & 8,3 \\
\hline plus-que-parfait & 0 & 0,0 & 0 & 0,0 & 0 & 0,0 & 0 & 0,0 \\
\hline FdB pour PC & 2 & 2,7 & 21 & 14,3 & 11 & 25,6 & 14 & 23,3 \\
\hline FdB pour IMP & 35 & $\mathbf{4 7 , 9}$ & 13 & 8,8 & 4 & 9,3 & 0 & 0,0 \\
\hline total & 73 & 100 & 147 & 100 & 43 & 100 & 60 & 100 \\
\hline
\end{tabular}

Tableau 9. Les modes d'action et leurs temps préférés en ESP FR L2

Dans le corpus NL FR L2 (cf. tableau 8), l'état favorise l'apparition de l'IMP 23, qui l'emporte aussi dans le cas des trois autres modes d'action. Les apprenants associent également l'état avec une forme au présent et très exceptionnellement avec un PC ou un présent à valeur de PC. Quant aux verbes d'activité et d'accomplissement, l'IMP est de nouveau privilégié mais dans une moindre mesure 24 ; ces modes d'action déclenchent dans $\pm 20 \%$ des cas le PC 25-26 et le présent à valeur de PC. Pour l'achèvement, l'IMP partage la scène avec le PC 27.

23. Mais il ne veut pas tout laisser comme ça. [4.20] (vouloir : verbe d'état) 
24. «No, mon dieu»: disait-il: ce n'est pas possible!» [4.18] (dire: verbe d'accomplissement)

25. Ben, ils ont célibré leur convaint. [4.3] (célébrer : verbe d'activité)

26. Mon père a immédiatement pris sa longue-vue et $a$ aussi vu le pavillon avec la tête de mort. [4.12] (voir + objet délimité : verbe d'accomplissement)

27. Mais son grand-père, normalement toujours le héro, a perdu. [4.19] (perdre : verbe d'achèvement)

En ESP FR L2 (cf. tableau 9), la situation est totalement différente : l'état s'exprime avant tout à l'aide de verbes conjugués au présent et dans une moindre mesure au PC 28. Lorsque l'IMP est employé, il l'est le plus souvent avec des verbes d'état 29. Pour les trois autres modes d'action, le PC apparaît dans deux tiers des occurrences 303132. Ces résultats ne sont pas sans rappeler le sous-emploi généralisé de l'IMP d'une part et les propriétés du stade acquisitionnel d'autre part, stade que nous avons défini comme étant post-basique.

28. Un navegateur qui $a$ èté sur le mât a vu un bateau sur la mer. [3.3] (être : verbe d'état)

29. Il se l'a dit qu capitaine et il a pris sa longue-vue pour voir que c'était la chose qui avait dans la distance. [3.1] (être, avoir : verbe d'état)

30. Les pirates ont célébré leur victoire. [3.1] (célébrer : verbe d'activité)

31. Un membre de l'équipag a vu autre bateau, il a averti au capitaine. [3.4] (voir + objet délimité : verbe d'accomplissement)

32. Finalement le pirate a attaché le capitaine au mât. [3.1] (attacher : verbe d'achèvement)

\subsection{Bilan}

Arrivées au terme de cette analyse, nous aimerions reprendre nos questions et hypothèses de recherche.

\section{Les apprenants emploient-ils les temps du passé dans leurs narrations et si oui, lesquels?}

Les deux groupes d'apprenants sont capables d'utiliser les moyens morphologiques pour référer au passé. Ils exploitent tous le passé composé et l'imparfait mais ils recourent aussi au présent à valeur de passé (dans un quart des occurrences en NL FR L2 et dans un tiers en ESP FR L2). L'emploi plus récurrent du présent par les hispanophones constitue un indice de leur niveau moins avancé, ce qui se trouve par ailleurs confirmé par l'absence du plus-que-parfait (temps apparaissant déjà occasionnellement dans le corpus NL FR L2). De plus, les deux corpus se distinguent par le choix préférentiel parmi les temps du passé : chez les néerlandophones il existe une tendance à surgénéraliser l'imparfait alors que chez les hispanophones c'est le passé composé qui l'emporte. Ces cas de suremploi concernent fréquemment des emplois fonctionnellement incorrects.

Pour ce qui est de l'hypothèse associée concernant le rôle de l'imparfait dans les deux corpus, elle se trouve confirmée dans le cas des néerlandophones (suremploi) mais non dans le cas des hispanophones (évitement) chez qui nous nous étions attendues à un emploi approprié de ce temps verbal.

\section{Les apprenants associent-ils correctement temps verbal et expression temporelle de type adverbial?}

Les expressions temporelles sont quasi absentes du corpus ESP FR L2; en revanche, nous y avons observé un emploi régulier du connecteur et pour établir des rapports chronologiques de type successivité immédiate. Ce et est également attesté dans le corpus NL FR L2 où figurent tout de même 91 expressions temporelles. Dans la plupart des cas, celles-ci épousent bien la valeur véhiculée par le temps verbal, sauf pour l'imparfait, temps pour lequel nous avons relevé plus de combinaisons «IMP + TA » incorrectes que correctes.

L'hypothèse associée à cette question qui prédisait l'existence de couples «temps + TA » appropriés se trouve infirmée par nos données.

\section{Les apprenants s'appuient-ils avant tout sur le mode d'action pour choisir un temps verbal?}


Dans le corpus NL FR L2 tous les modes d'action s'expriment de préférence par l'imparfait. Seul dans le cas de l'achèvement il subit une réelle concurrence du passé composé. Pour l'accomplissement le passé composé vient en deuxième position alors qu'avec l'activité et l'état la deuxième forme la plus fréquente est le présent. Chez les apprenants hispanophones, par contre, c'est d'une part le passé composé qui prédomine avec des verbes d'achèvement, d'accomplissement et d'activité et d'autre part le présent qui l'emporte avec des verbes d'état. Pour ces derniers le deuxième temps employé est l'imparfait.

Notre hypothèse associée est donc partiellement vérifiée : les modes d'action jouent un rôle dans la sélection du temps verbal. Toutefois, les mécanismes de sélection ne sont pas les mêmes auprès des deux groupes d'apprenants.

Dans les parties suivantes, nous proposerons quelques pistes d'explication possibles pour les tendances qui caractérisent les deux corpus.

\section{Hypothèse de la primauté de l'aspect lexical}

Pour expliquer les résultats relevés en $(\$ 4.1)$ et en $(\$ 4.2)$, il nous a semblé intéressant de vérifier en $(\$ 4.3)$ si les apprenants se laissent guider par le mode d'action dans leur choix du temps verbal, ce qui confirmerait l'hypothèse de la primauté de l'aspect lexical (HPAL, cf. Andersen \& Shirai 1996). L'HPAL prévoit que les apprenants FLE utilisent d'abord le PC, marqueur de l'aspect perfectif en français, avec des verbes téliques (les verbes d'accomplissement et d'achèvement) et qu'ils étendent petit à petit l'emploi de cette forme verbale aux autres classes aspectuelles lexicales (les verbes d'activité et d'état). De plus, elle postule que l'IMP, marqueur de l'aspect imperfectif, apparaît plus tard que le PC en combinaison avec des verbes atéliques et qu'il élargit son emploi progressivement vers les verbes téliques.

Confrontées avec d'importants écarts entre nos deux groupes d'apprenants, nous n'avons pas pu vérifier l'HPAL. Afin de mieux la cerner, il nous semble utile de comparer nos corpus d'apprenants au corpus de contrôle francophone. Dans ce qui suit, nous ne tiendrons compte que des formes réalisées de l'imparfait et du passé composé. Nous écartons le plus-que-parfait, faute d'exemples dans le corpus ESP FR L2, ainsi que le présent qui dans le corpus des natifs prend la valeur du présent historique et qui ne fonctionne donc pas parallèlement aux présents dans les corpus d'apprenants.

\begin{tabular}{|l|c|c|c|c|c|c|c|c|c|c|c|c|}
\hline chiffres & \multicolumn{4}{|c|}{ état } & \multicolumn{3}{c|}{ activité } & \multicolumn{3}{c|}{ accomplissement } & \multicolumn{3}{c|}{ achèvement } \\
\hline & NL & ESP & & NL & ESP & & NL & ESP & & NL & ESP \\
& FR & FR & & FR & FR & & FR & FR & & FR & FR \\
& L2 & L2 & FR L1 & L2 & L2 & FR L1 & L2 & L2 & FR L1 & L2 & L2 & FR L1 \\
& 8 & 24 & 22 & 28 & 102 & 22 & 15 & 27 & 14 & 65 & 41 & 64 \\
\hline PC & 132 & 12 & 31 & 60 & 11 & 22 & 40 & 1 & 1 & 66 & 5 & 11 \\
\hline IMP & 140 & 36 & 53 & 88 & 113 & 44 & 55 & 28 & 15 & 131 & 46 & 75 \\
\hline
\end{tabular}

Tableau 10. Les modes d'action et leurs temps préférés en NL-ESP FR L2 et en FR L1

\begin{tabular}{|l|c|c|c|c|c|c|c|c|c|c|c|c|}
\hline$\%$ & \multicolumn{3}{|c|}{ état } & \multicolumn{3}{c|}{ activité } & \multicolumn{3}{c|}{ accomplissement } & \multicolumn{3}{c|}{ achèvement } \\
\hline & $\begin{array}{c}\text { NL } \\
\text { FR L2 }\end{array}$ & $\begin{array}{c}\text { ESP } \\
\text { FR L2 }\end{array}$ & FR L1 & $\begin{array}{c}\text { NL } \\
\text { FR L2 }\end{array}$ & $\begin{array}{c}\text { ESP } \\
\text { FR L2 }\end{array}$ & FR L1 & $\begin{array}{c}\text { NL } \\
\text { FR L2 }\end{array}$ & $\begin{array}{c}\text { ESP } \\
\text { FR L2 }\end{array}$ & FR L1 & $\begin{array}{c}\text { NL } \\
\text { FR L2 }\end{array}$ & $\begin{array}{c}\text { ESP } \\
\text { FR L2 }\end{array}$ & FR L1 \\
\hline PC & 5,7 & 66,7 & 41,5 & 31,8 & 90,3 & 50,0 & 27,3 & 96,4 & 93,3 & 49,6 & 89,1 & 85,3 \\
\hline IMP & 94,3 & 33,3 & 58,5 & 68,2 & 9,7 & 50,0 & 72,7 & 3,6 & 6,7 & 50,4 & 10,9 & 14,7 \\
\hline
\end{tabular}


Tableau 11. Les modes d'action et leurs temps préférés en NL-ESP FR L2 et en FR L1

D'après l'HPAL, il existe un itinéraire acquisitionnel qui va du stade (i) à (iv) (cf. infra). Comme nous ne faisons pas d'étude longitudinale, nous ne sommes pas en mesure de vérifier ce parcours mais il nous est toutefois possible de déterminer si les natifs se situent bien au niveau (iv), caractérisé par une polyvalence de l'IMP et du PC, et si les apprenants se rapprochent de ce stade final.

i. PC pour accomplissement + achèvement

ii. PC pour état et activité

iii. IMP pour état

iv. IMP pour les autres modes d'action

Du tableau 11, il ressort clairement que les quatre modes d'action se réalisent par les deux temps dans les trois corpus mais pas avec le même degré d'intensité. Ainsi en FR L1, l'opposition PC-IMP est la plus marquée avec les verbes d'accomplissement; les apprenants ESP s'alignent sur ce modèle, contrairement aux apprenants NL. Nous relevons un phénomène analogue avec les verbes d'achèvement pour lesquels les apprenants NL recourent tout de même un peu plus souvent au PC. En ce qui concerne les verbes d'activité, les hispanophones s'écartent plus du modèle FR L1 que les néerlandophones et l'inverse se produit pour les verbes d'état. Sur base de ces résultats, nous pourrions donc affirmer que les apprenants ESP ont certainement atteint les niveaux (i) et (ii) et que le stade (iii) est en voie de réalisation. Pour les néerlandophones, l'image est un peu plus nuancée : à partir de leur emploi de l'imparfait, nous aurions tendance à leur assigner le niveau (iv) mais si nous prenons en considération le taux plus bas du PC pour l'état et surtout pour l'accomplissement, il est difficile de maintenir ce niveau (iv). Afin d'expliquer ce paradoxe, nous examinerons en (\$6) l'hypothèse du transfert.

\section{Hypothèse du transfert}

L'hypothèse du transfert pourrait être un outil explicatif performant dans le domaine de l'emploi des temps du passé, d'autant plus que, dans notre cas, il existe un écart considérable entre les langues étudiées quant à l'encodage de la référence au passé. Là où les aspects perfectifs et imperfectifs s'expriment par l'imperfectum en néerlandais, le français et l'espagnol disposent d'un système verbal qui marque par des moyens morphologiques différents tantôt l'aspect grammatical perfectif (le passé composé et le passé simple) tantôt l'aspect grammatical imperfectif (l'imparfait).

\begin{tabular}{|l|c|c|c|c|}
\hline Emploi des temps \% & NL FR L2 & NL L1 & ESP FR L2 & ESP L1 \\
\hline PC (PS en FR L1/ESP L1) & $\mathbf{1 8 , 8}$ & 0 & 56,6 & 58,6 \\
\hline IMP & 48,3 & $\mathbf{8 1 , 4}$ & 8,5 & 17,1 \\
\hline PR & $\mathbf{2 5 , 3}$ & 8,8 & $\mathbf{3 3 , 5}$ & 19,8 \\
\hline PQP & 5,5 & 8,8 & 0 & 2,7 \\
\hline autres & 2,1 & 1 & 6,8 & 1,8 \\
\hline
\end{tabular}

Tableau 12. Emploi des temps en FR L2 par des apprenants NL et ESP et en NL et ESP L1

La forme verbale du passé est clairement l'imperfectum en néerlandais, avec 158 occurrences pour l'ensemble des huit textes, ce qui revient à $81,4 \%$. En second lieu viennent le plusquamperfectum et le praesens avec chacun $8,8 \%$. Le perfectum n'est pas attesté dans le corpus.

D'après ces résultats, il est clair que le suremploi de l'IMP dans le corpus NL FR L2 relève d'une interférence de la L1 et que l'hypothèse du transfert se confirme : il s'agit d'un transfert que nous pouvons qualifier de « négatif ». Pourtant, les apprenants NL ont déjà une certaine conscience du système de la langue cible car dans leur interlangue apparaissent des PC tout comme des présents à valeur de PC. 
Quant aux apprenants hispanophones, nous sommes plutôt en présence d'un mécanisme de transfert «positif» en devenir : ils pourraient profiter des ressemblances entre leurs langues source et cible mais comme ils se situent à un niveau moins avancé que les apprenants NL, leur emploi de l'imparfait est encore défaillant: ils évitent en réalité la forme, morphologiquement complexe au niveau de la conjugaison.

\section{Conclusion et perspectives didactiques}

Malgré le caractère préliminaire de notre étude consacrée à l'expression de la temporalité par des apprenants FLE, un certain nombre de tendances intéressantes ont pu être dégagées. Ainsi, nos deux groupes d'apprenants ne montrent pas les mêmes préférences pour la référence au passé : mis à part le présent, les néerlandophones préfèrent l'imparfait et les hispanophones le passé composé mais, lorsque les néerlandophones emploient le passé composé, ils le font de manière plus correcte que les hispanophones. Nous avons tenté d'expliquer ces deux phénomènes de manière interne, à l'aide d'une étude des expressions temporelles et des modes d'action. Pour ce qui est du premier critère, les apprenants NL interprètent correctement la valeur du PC puisqu'ils sont capables d'associer adéquatement le $\mathrm{PC}$ à une expression temporelle. En revanche, avec l'imparfait ils se trompent plus facilement. Les apprenants ESP emploient trop peu d'expressions temporelles pour avancer des conclusions : ils semblent plus se concentrer sur l'essentiel des événements qu'ils relient éventuellement entre eux à l'aide du connecteur et. L'étude des modes d'action confirme la non-intégration du système temporel français dans l'interlangue des néerlandophones d'une part et révèle un système en devenir d'autre part : avec le mode d'action le plus marqué, l'achèvement (télique, dynamique et ponctuel), le PC est privilégié. L'étude de corpus de contrôle en L1 a confirmé que les hispanophones paraissent profiter des analogies entre leur L1 et la L2. Ils bénéficient d'un transfert positif alors que les néerlandophones sont victimes d'un transfert négatif qui débouche sur un suremploi de l'imparfait.

L'intérêt de notre étude réside en l'approche pluridimensionnelle de la référence au passé dans deux corpus d'apprenants et trois corpus de contrôle. Toutefois, ces corpus utilisés sont relativement restreints et donc non représentatifs d'une population. Il est par conséquent nécessaire d'augmenter le nombre d'apprenants et de locuteurs natifs pour réaliser une analyse statistique exhaustive.

Quant au processus d'apprentissage même, il serait intéressant d'étudier l'effet de l'instruction de la temporalité en FRL2. D'après nos résultats, il semblerait que l'enseignement grammatical explicite, dont nos apprenants ont bénéficié, porte ses fruits. Certains chercheurs tels que Cadierno (1995) et Hendrix et alii (2002) se sont concentrés sur l'optimisation de l'efficacité de l'instruction basée sur l'input et sur l'intake qu'il déclenche. La figure ci-dessous, inspirée de Van Patten (1992 cité par Cadierno 1995 : 180), représente la différence entre «traditional instruction», qui se focalise sur la production des formes verbales dans l'output, et "processing instruction», qui se concentre sur la compréhension et l'interprétation des temps verbaux.

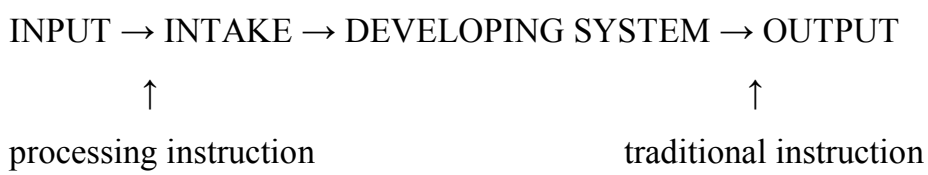

Figure 1. Le modèle acquisitionnel d'une langue seconde de VanPatten

Afin d'optimaliser l'intake, il faudrait que les apprenants parviennent à établir les bonnes connexions entre la forme et la fonction. Selon Hendrix et alii (2002: 75), l'input proposé de manière déductive s'avérerait très efficace pour réaliser cet objectif : « l'enseignement part de la description grammaticale de la structure cible pour en déduire l'interprétation des occurrences présentes dans l'input ». Il serait intéressant de creuser ce type d'instruction à plus large échelle et d'en vérifier l'opérationnalité didactique dans le domaine de l'acquisition de la temporalité en FR L2. 


\section{Références bibliographiques}

Amenós Pons, J. (2010). Los tiempos de pasado del español y el francés : semántica, pragmática y aprendizaje de E/EL : perspectivas desde la Teoría de la Relevancia. Thèse de doctorat inédite. Universidad Nacional de Educación a Distancia (España).

Andersen, R.W. (1991). Developmental sequences: The emergence of aspect marking in second language acquisition. In Huebner, T. \& Ferguson, C. A. (éds), Crosscurrents in second language acquisition and linguistic theories, Amsterdam, Benjamins, 305-324.

Andersen, R. W. \& Shirai, Y. (1996). Primacy of aspect in first and second language acquisition: The pidgin/creole connection. In Ritchie, W.C. \& Bhatia, T.K. (éds), Handbook of second language acquisition, San Diego, CA: Academic Press, 527-570.

Bartning, I. \& Schlyter, S. (2004). Itinéraires acquisitionnels et stades de développement en français L2. In Myles, F. \& Towel, R. (éds), The acquisition of French as a second language. (French Language Studies 14, Special Issue), Cambridge University Press, 281-299.

Benazzo, S. \& Starren, M. (2007). L'émergence de moyens grammaticaux pour exprimer les relations temporelles en L2. Acquisition et interaction en langue étrangère, 25 : 129-158.

Bergström, A. (1995). The expression of temporal reference by English speaking learners of French, Thèse de Doctorat, Pennsylvania State University.

Cadierno, T. (1995). Formal instruction from a processing perspective: An investigation into the Spanish past tense. The Modern Language Journal 79, 179-193.

Delatour, Y. et alii (1991). Grammaire du français, Paris, Hachette.

Giacalone-Ramat, A. (2002). How do learners acquire the classical three categories of temporality?. In Salaberry, R. \& Shirai, Y. (éds), The L2 acquisition of tense-aspect morphology, Amsterdam-Philadelphia, Benjamins, 221247.

Gosselin, L. (1996). Sémantique de la temporalité en français. Un modèle calculatoire et cognitif du temps et de l'aspect, Louvain-la-Neuve, Duculot.

Gosselin, L. \& François, J. (1991). Les typologies de procès. Des verbes aux prédications. In Fuchs, C. (éd.), Les typologies de procès, Strasbourg, Klincksieck, 19-86.

Hendrix, L., Housen, A. \& Pierrard, M. (2002). Mode d'implémentation de l'instruction grammaticale explicite et appropriation de langues étrangères, Acquisition et interaction en langue étrangère 16, en ligne, URL : http://aile.revues.org/1394.

Hopper, P.J. (1979). Aspects of foregrounding in discourse. In Givón, T. (éd.), Syntax and semantics, vol. 12, New York, Academic Press, 213-241.

Housen, A. (2002). The development of tense-aspect in English as a second language and the variable influence of inherent aspect. In Salaberry, R. \& Shirai, Y. (éds), The L2 acquisition of tense-aspect morphology, AmsterdamPhiladelphia, Benjamins, 155-197.

Howard, M. (2002). L'acquisition des temps du passé en français par l'apprenant dit avancé : une approche lexicale. In Labeau, E. \& Larrivée, P. (éds), Les temps du passé français et leur enseignement. Amsterdam-New York, Editions Rodopi B.V, 181-204.

Howard, M. (2012). The Development of Verb Morphology in the Advanced Learner Variety: A Longitudinal Study of L2. French Chinese Journal of Applied Linguistics 35/2, 153-174.

Imbs, P. (1960). L'emploi des temps verbaux en français moderne. Paris, Klincksieck.

Izquierdo, J. (2009). L'aspect lexical et le développement du passé composé et de l'imparfait en français L2 : Une étude quantitative auprès d'apprenants hispanophones. La revue canadienne des langues vivantes 65/4, 587-613.

Izquierdo, J. \& Collins, L. (2008). The facilitative effects of L1 influence on L2 tense-aspect marking: Hispanophones and Anglophones learning French. The Modern Language Journal 93, 350-368. 
Judge, A. (2002). Écarts entre manuels et réalités : un problème pour l'enseignement des temps du passé à des étudiants d'un niveau avancé. In Labeau, E. \& Larrivée, P. (éds), Les temps du passé français et leur enseignement (Cahiers Chronos 9), 135-156.

Kihlstedt, M. (1998). La référence au passé dans le dialogue. Etude de l'acquisition de la temporalité chez des apprenants dits avancés de français. Thèse de doctorat, Stockholm.

Klein, W. (1994). Time in language. London-New York, Routledge.

Labelle, M. (1987). L'utilisation des temps du passé dans les narrations françaises : le passé composé, l'imparfait et le présent historique. Revue Romane 22-1, 3-29.

Labeau, E. (2005). Beyond the aspect hypothesis. Tense-aspect development in advanced L2 French. Bern, P. Lang.

Lindgren, C. (2008), "Regarde, maman : le soleil se leva ». Emploi des temps du passé dans des récits d'enfants francophones en Suède. Upsala, ACTA UNIVERSITATIS UPSALIENSIS (Studia Romanica Upsaliensia 76).

Noyau, C. \& Vasseur, M.-T. (1986). L'acquisition des moyens de la référence temporelle en français langue étrangère chez des adultes hispanophones. Langages, 84 : 105-117.

Noyau, C. (1998). Le développement de la temporalité dans le récit : interactions entre processus de construction des formes et processus de construction de textes. Studi Italiani di Linguistica Teorica e Applicata 1, 111-132.

Paprocka-Piotrowska, U. (2002). Mais dans ce moment le chien est venu ou comment les apprenants formels polonophones s'approprient le système temporel du français. In Labeau, E. \& Larrivée, P. (éds), Les temps du passé français et leur enseignement. Amsterdam-New York, Editions Rodopi B.V, 205-229.

Populier, V. (1985). Les temps du passé. Analyse contrastive français-néerlandais. Mémoire de Licence, Université de Gand.

Reichenbach, H. (1966). Elements of symbolic logic. London-New York, Macmillan.

Salaberry, R. (2005). Evidence for transfer of knowledge of aspect from L2 Spanish to L3 Portuguese. In Ayoun, D. \& Salaberry, R. (éds), Tense and aspect in Romance languages, Amsterdam-Philadelphia, Benjamins, 179-210

Schlyter, S. (1996). Télicité, passé composé et types de discours dans l'acquisition du français langue étrangère. Revue française de linguistique appliquée $1 / 1,107-118$.

Starren, M. (2001). The second time. The acquisition of temporality in Dutch and French as a second language. Utrecht, LOT.

Stutterheim, Chr. von (1991). Narrative and description. In Huebner T. \& Ferguson C.A. (éds), Cross Currents in Second Language Acquisition and Linguistic Theory, Amsterdam-Philadelphia, Benjamins, 385-403.

Van der Linden, E. (2000). De verleden tijd in het Frans van Nederlanders. Invloed van input, interferentie en onderwijs. Leuvense Bijdragen 89/1-2, 231-250.

Vendler, Z. (1967). Verbs and times. In Vendler, Z. (éd.), Linguistics in philosophy, Ithaca, NY Cornell University Press, 97-121.

Vertriest, F. (2005), La référence au passé dans la narration écrite. Mémoire de licence, Université de Gand.

Weinrich, H. (1973). Le temps. Le récit et le commentaire. Paris, Seuil.

Wiberg, E. (1996). Reference to past events in bilingual Italian-Swedish children of school age. Linguistics 34, 10871114.

Wiberg, E. (2002). Reference to past and verticalization in advanced NNSs dialogues. Revue française de linguistique appliquée 7/2, 43-50.

Wilmet, M. (2007). Grammaire rénovée du français. Bruxelles, De Boeck.

\footnotetext{
${ }^{1}$ Il s'agit plus spécifiquement de la marque morphologique du temps verbal, des locutions adverbiales ou prépositionnelles de temps et des modes d'action. Nous ne traiterons pas ici de la distinction entre avant-plan et arrière-plan qui est étroitement liée à l'organisation de la phrase et aux modes de liaison entre prédications.
} 
${ }^{2}$ Les exemples de courir (verbe d'activité) et de courir cinq kilomètres (verbe d'accomplissement) illustrent, selon Labeau (2002), un point faible dans la répartition vendlerienne car la classification présupposerait l'existence de verbes homonymes. Wilmet (2007 : 71) admet en revanche que « la démarcation [entre types] est franchissable grâce aux compléments du verbe ».

${ }^{3}$ Comme il n'est pas toujours facile de distinguer les verbes d'achèvement des verbes d'accomplissement, certains chercheurs, tels que Klein (1994), Wiberg (1996) et Labeau (2005), les regroupent dans une seule classe de verbes téliques.

${ }^{4}$ Pour un aperçu des affinités et des incompatibilités entre le temps, l'aspect grammatical, le mode d'action et les compléments adverbiaux en français, voir Labeau (2005: 82-88).

${ }^{5}$ Les termes de premier plan et d'avant-plan seront utilisés de manière interchangeable.

${ }^{6}$ Nous faisons abstraction de la remarque sur le passé simple de Poisson-Quinton et alii (2002: p. 49): «On peut aussi l'employer dans un contexte présent, il donne alors une dimension historique aux faits. »

${ }^{7}$ En regroupant passé composé et passé simple, nous faisons abstraction du lien intrinsèque avec le MP dans le cas du passé composé. Le passé simple français n'a pas d'équivalent en néerlandais où il se trouve traduit, tout comme l'imparfait, par l'imperfectum.

${ }^{8}$ Nous verrons plus loin que les apprenants hispanophones sont moins avancés sur l'itinéraire acquisitionnel que les néerlandophones.

${ }^{9}$ Cf. Andersen \& Shirai (994: 133, cité par Starren $2001: 80$ ) : « [F]irst and second language learners will initially be influenced by the inherent semantic aspects of verbs or predicates in the acquisition of tense and aspect markers associated with or affixed to these verbs. »

${ }^{10}$ En Flandre, l'enseignement du français commence déjà dans la $5^{\text {ème }}$ année de l'enseignement primaire, quand les élèves ont en principe entre 10 et 11 ans. En quatrième année, ils devraient avoir atteint le niveau intermédiaire. En Espagne, le français constitue une matière optionnelle dans l'enseignement secondaire. En troisième année, les élèves devraient avoir atteint le niveau post-initial (cf. Bartning \& Schlyter 2004 pour une description des stades acquisitionnels).

${ }^{11}$ Afin de faciliter le travail, les mots suivants ont été mis au tableau : le capitaine Hadoque, un navire, une vigie, une longue-vue, un pavillon, une tête de mort, un canon, une hache, une épée, un pistolet, attacher à un pilier, la pleine lune, ivre.

${ }^{12}$ Les corpus FR L2 par des néerlandophones, FR L1 et NL L1 ont été réunis par Fransiska Vertriest, étudiante à l'Université de Gand, dans le cadre d'un mémoire de licence en langues et littératures romanes. Nous la remercions pour la mise à notre disposition de ses données. Ariane Ruyffelaert a récolté les narrations en FR L2 par des hispanophones et en espagnol L1.

${ }^{13}$ [4.11] désigne le corpus (4 pour le corpus NL FR L2 et 3 pour le corpus ESP FR L2), 11 identifie à l'élève.

${ }^{14} \mathrm{Il}$ s'agit de quelques rares exemples de futur et de conditionnel.

${ }^{15}$ Il aurait pu être «présent historique », emploi souvent attesté dans des narrations de locuteurs natifs pour référer à des événements passés. Le présent historique y alterne généralement avec le passé simple. Or, comme les apprenants utilisent cette forme également dans le domaine de l'arrière-plan, où sa présence n'est pas usuelle, nous ne la considérons pas comme illustrative du présent historique.

${ }^{16}$ Ces erreurs sont essentiellement dues à une surgénéralisation de ces temps. 